\title{
Editorial
}

\section{Advanced Modeling in Stochastic Computational Electromagnetics}

\author{
Dragan Poljak (D), ${ }^{1}$ Flavia Grassi $\mathbb{D}^{2},{ }^{2}$ Milica Rančić, ${ }^{3}$ and Sergey Tkachenko $\mathbb{D}^{4}$ \\ ${ }^{1}$ University of Split, Split, Croatia \\ ${ }^{2}$ Politecnico di Milano, Milano, Italy \\ ${ }^{3}$ Mälardalen University, Västerås, Sweden \\ ${ }^{4}$ Otto-von-Guericke Universität, Magdeburg, Germany
}

Correspondence should be addressed to Dragan Poljak; dpoljak@fesb.hr

Received 11 June 2018; Accepted 11 June 2018; Published 9 July 2018

Copyright (C) 2018 Dragan Poljak et al. This is an open access article distributed under the Creative Commons Attribution License, which permits unrestricted use, distribution, and reproduction in any medium, provided the original work is properly cited.

The tremendous development of digital computers in the last decades has provided continuous improvement of various sophisticated methods in computational electromagnetics (CEM). CEM analysis methods provide results within an appreciably shorter time than it would be required to build and test the corresponding prototype by means of certain experimental procedures. Furthermore, computer simulation of electromagnetic phenomena enables one to predict a system behavior for a wide range of parameters such as geometry, material properties, boundary and initial conditions, and excitations. Limitations of CEM models when applied to complex configurations resulting in large-scale problems are often related to very high computational cost.

On the other hand, significant discrepancies between calculated and measured results mainly stem due to uncertainties in an input data set arising from numerical instabilities, geometry, manufacturing defects, noise in measurements, and unpredictable and uncontrollable environmental effects. Therefore, of crucial interest for CEM models is to quantify uncertainty propagation due to various sources of uncertainty, as well as to obtain a meaningful statistics of the target variables by resorting to a limited number of simulations.

A plausible and efficient measure of these uncertainties in a set of inputs is one of the stochastic methods. Namely, stochastic or stochastic-deterministic modeling can be applied with the objective to quantify such uncertainties by providing one or more output parameters, as random variables, and by computing corresponding statistical moments.
In particular, stochastic problems could be divided into two groups: the first one dealing with deterministic system and stochastic fields and the second one when the parameters of the system are unknown.

This special issue aims to invite prospective authors to submit full-length journal papers covering all aspects of stochastic computational electromagnetics. All submitted manuscripts will be subjected to the peer review process and will be considered for publication in the special issue.

Therefore, the Guests Editors enthusiastically accepted the opportunity to edit a special issue covering some important aspects of stochastic computational electromagnetics.

The issue starts with a paper entitled "Stochastic Collocation Applications in Computational Electromagnetics", by D. Poljak et al. This paper reviews the application of Stochastic Collocation (SC) technique in electromagnetic compatibility and bioelectromagnetics.

The paper by N. Toscani et al. entitled "A Possibilistic Approach for the Prediction of the Risk of Interference between Power and Signal Lines Onboard Satellites" deals with a hybrid random/fuzzy approach for uncertainty quantification in electromagnetic modeling, which combines probability and possibility theory in order to properly account for both aleatory and epistemic uncertainty, respectively.

The paper "Propagation of Current Waves along Randomly Located Multiconductor Transmission Lines inside a Rectangular Resonator", written by S. V. Tkachenko et al., 
deals with the propagation of current waves along stochastic transmission lines inside a resonator. The paper "Anticollision Method of Active Magnetic Guidance Ranging for Cluster Wells" by X. Dou et al. presents the active magnetic guidance ranging method for cluster wells.

The paper entitled "A Kind of Stochastic Eigenvalue Complementarity Problems", by Y. Wang and S. Du, proposes a new type of stochastic eigenvalue complementarity problem and the smoothing Newton method to treat the problem, as well.

The paper "Reliable Approach for EM Scattering Calculation from Sea Surface Covered with Foams Based on MFBM and VRT Theory", by T. Song et al., yields a reliable approach based on modified facet-based model and Vector Radiative Transfer theory to calculate electromagnetic scattering from a particular electrically large sea surface superimposed with foams.

Finally, the paper "A New Shooting Bouncing Ray Method for Composite Scattering from a Target above the Electrically Large Scope Sea Surface", by P. Peng and G. Lixin, deals with a new shooting and bouncing ray (SBR) simulator based on the hybrid scheme of GO/PO/SDFM/EEC method for the accurate prediction of composite scattering from a low altitude target above the electrically very-large-scale sea surface.

The Guest Editors warmly hope they managed to put together an interesting piece of work regarding the use of stochastic models in computational electromagnetics.

\section{Acknowledgments}

The Guest Editors would like to thank all contributors for their valuable contributions and to the reviewers for their prompt response and excellent work, as well.

Dragan Poljak

Flavia Grassi

Milica Rančić

Sergey Tkachenko 


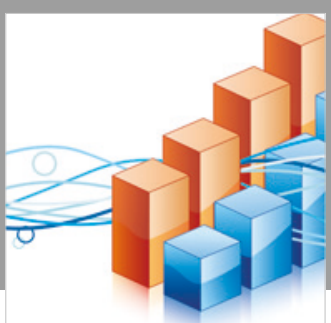

Advances in

Operations Research

\section{-n-m}
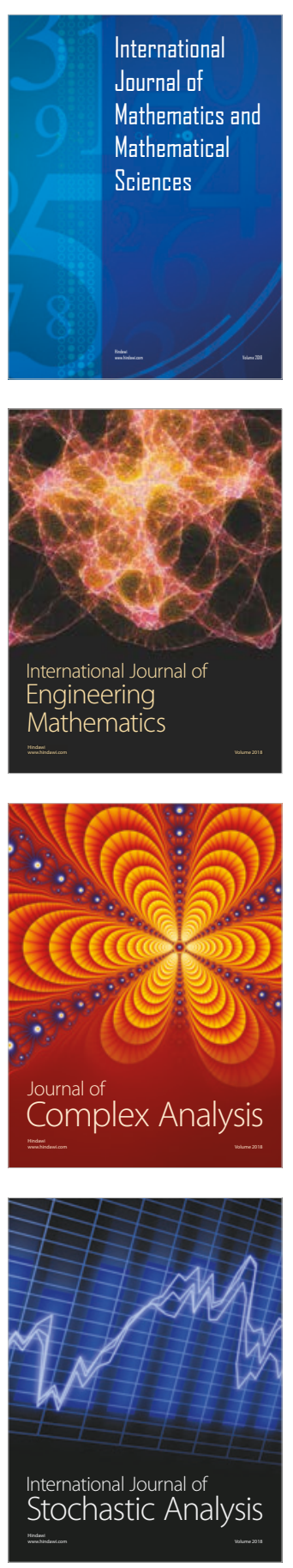
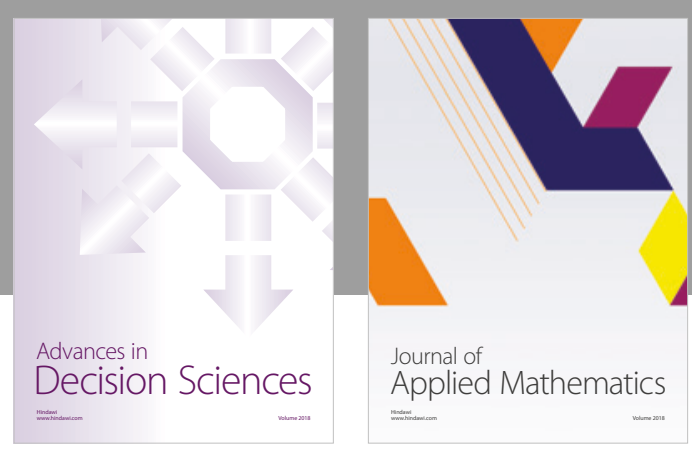

Journal of

Applied Mathematics
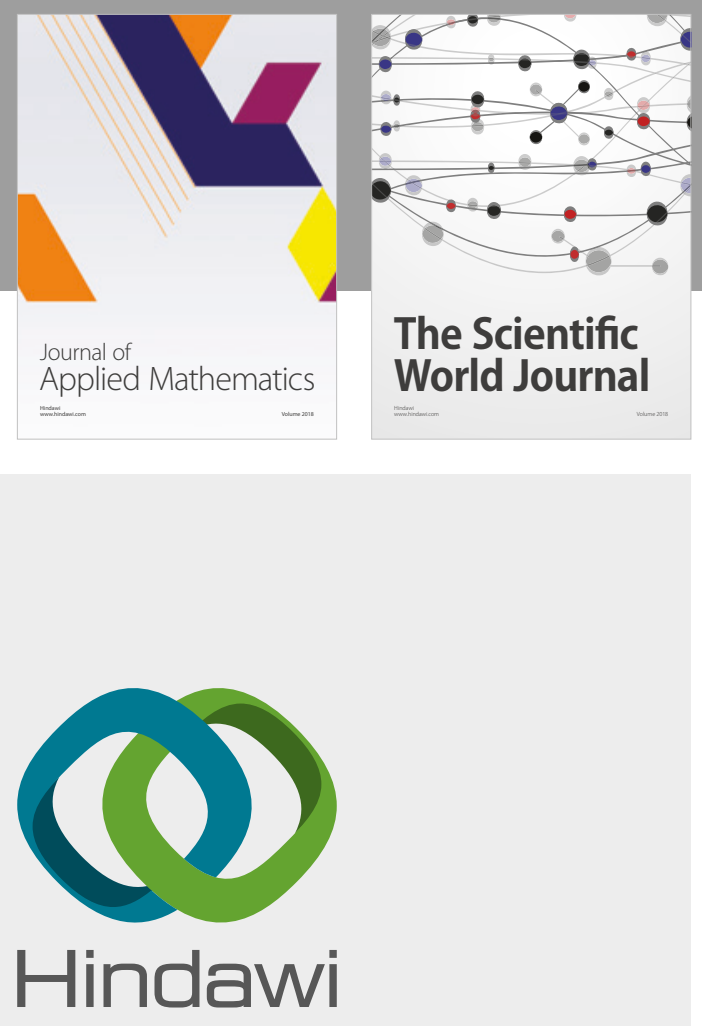

Submit your manuscripts at

www.hindawi.com

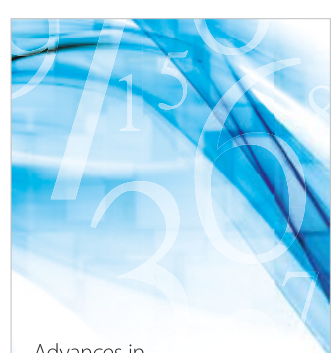

Advances in
Numerical Analysis
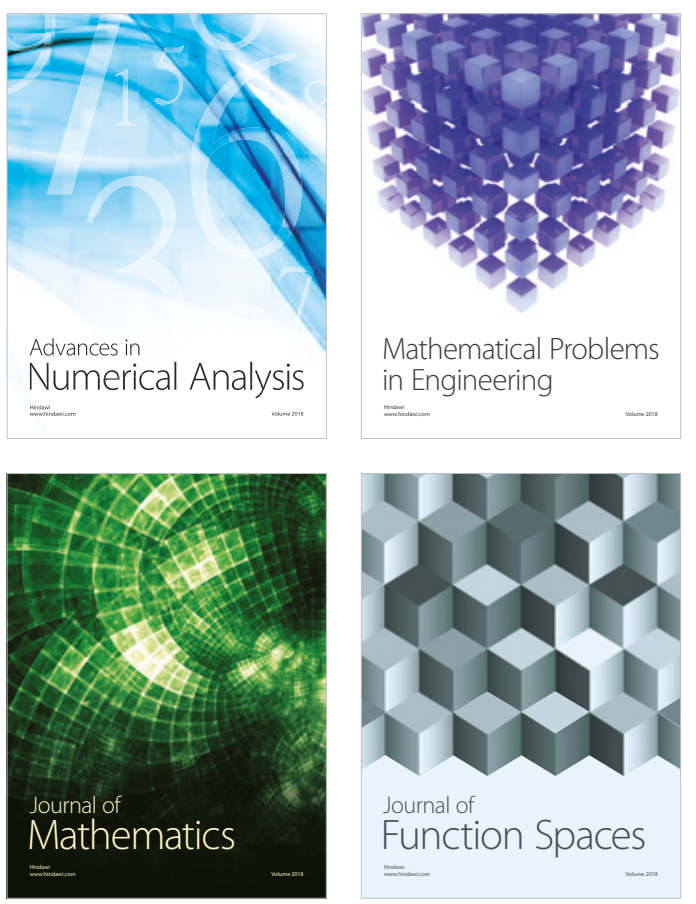

Mathematical Problems in Engineering

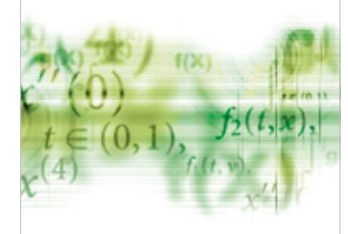

International Journal of

Differential Equations

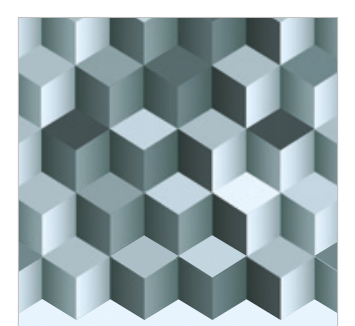

Journal of

Function Spaces

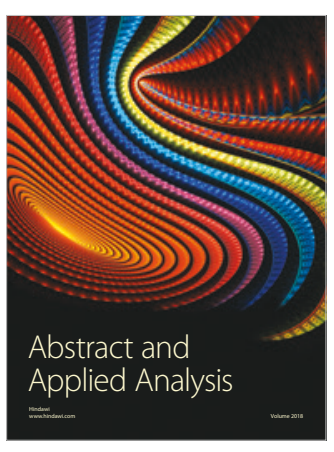

The Scientific

World Journal

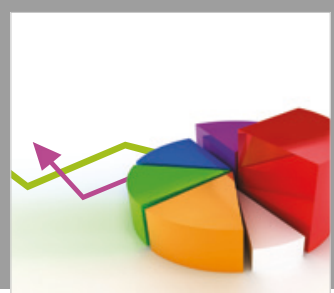

Journal of

Probability and Statistics
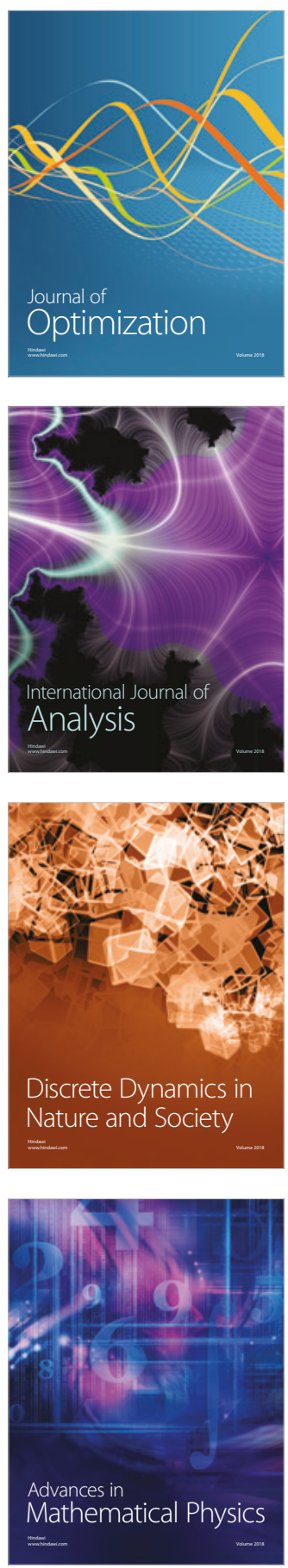